\title{
Clinical outcomes of COVID-19 in hemodialysis patients in the city of Zonguldak, Turkey
}

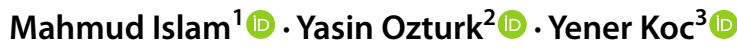

Received: 14 July 2020 / Accepted: 2 December 2020 / Published online: 15 January 2021

(c) The Author(s), under exclusive licence to Springer Nature B.V. part of Springer Nature 2021

\begin{abstract}
Purpose Patients on maintenance hemodialysis are vulnerable to viral infections like COVID-19 because of the low chance of obedience and complying with rules besides the need for transfer to distant dialysis facilities. We investigated the impact and clinical effect of treatment of COVID-19 in dialysis patients.

Methods We included patients on maintenance hemodialysis at different healthcare facilities in Zonguldak city. With the diagnosis of the first infected HD case, all other patients who shared the same session were screened. Hospitalized and clinically confirmed cases were included. COVID-19 diagnosis was made based on clinical, biochemical along radiologic findings. Results 34 (F/M:19/15, mean age $62 \pm 13.2$ years, dialysis duration $66.9 \pm 57.7$ months, length of hospital stay $16.2 \pm 7.9$ days) were diagnosed with COVID-19 infection. The prevalence of COVID-19 was found to be $18.4 \%$ of our exposed population. $38.2 \%$ of our patients were initially diagnosed by CT screening while asymptomatic. $35.3 \%$ had a fever as the first presenting symptom. Lymphopenia was the most common laboratory finding. Except for one, all had at least one comorbidity. Out of $12(35.3 \%)$ patients admitted to ICU 6(17.6\%) died. The deceased patients were older, presented with lower serum albumin and lymphocyte count, and had higher CRP and fibrinogen levels. High CRP level on admission was the only significant predictor of mortality.

Conclusion Early detection will lower mortality. In this study, with a low prevalence of COVID-19, the importance of early screening of both symptomatic and asymptomatic patients was shown to be highly important. Further studies are still needed to find out the most appropriate medical management.
\end{abstract}

Keywords COVID-19 $\cdot$ Hemodialysis $\cdot$ SARS-CoV-2 $\cdot$ Chronic renal failure $\cdot$ Filiation

\section{Introduction}

The World Health Organization (WHO) declared novel coronavirus disease (COVID-19) as a global pandemic in March 2020. COVID-19 is rapidly affecting the delivery of healthcare worldwide [1-3]. It is believed that COVID-19 is highly contagious and has particularly high morbidity in the elderly especially in those with comorbidities [1-5].

Mahmud Islam

drisleem@gmail.com

1 Department of Nephrology, Zonguldak Ataturk State Hospital, Nefroloji klinik, Huzur sokak, 67100 Zonguldak, Turkey

2 Zonguldak Bulent Ecevit University, Zonguldak, Turkey

3 Division of Nephrology, Department of Internal Medicine, Sivas Cumhuriyet University Medical Faculty, Sivas, Turkey
The transmission routes include droplet inhalation, direct person-to-person transmission through coughing and sneezing, and with contact of oral, nasal, and eye mucous membranes [6].

Many of the most common symptoms are somehow similar to those of seasonal flu and to a less extent common cold. The WHO has described fever and dry cough as the most common symptoms of COVID-19, nearly affecting 90 and $70 \%$ of infected patients respectively [7]. When the majority of alveolar ciliated cells are affected, they stop carrying out their main activity of clearing the airways resulting in sequential progressive accumulation of debris and fluids in the lungs. This may lead to a clinical picture of acute respiratory distress syndrome [6].

Chronic kidney disease(CKD) is a severe medical entity with a high prevalence of comorbid conditions, mostly including diabetes and cardiovascular disease, disproportionately affecting older adults [8]. Chronic Kidney disease 
is associated with an increased risk of both inpatient and outpatient pneumonia [9]. T-cell immunity plays a key role in recovery from COVID-19 infection [10]. Uremia is invariably associated with extensive impairment of both lymphocyte and granulocyte cell function.

The information regarding the pandemic of COVID-19 in hemodialysis (HD) patients is limited. This group is mobile, frequently traveling from home to dialysis centre and also to other healthcare facilities, serving as potential vectors. Fort that, it is more difficult to prevent and control highly contagious infections as the case in the general population [11-13]. Many HD patients are elderly, with more than one comorbidity, mainly cardiovascular with hypertension, diabetes, lung disease accompanied with underlying immune compromisation making them more vulnerable to worse outcomes in case of COVID-19 added on top [14].

Zonguldak is located in the western Black Sea region of Turkey. It is one of the most important coal mine cities where the incidence of occupational lung disease and COPD is reported to be high [15].

In our study, we aimed to determine the frequency, symptoms, effect of medical management, and clinical outcomes of COVID-19 infection among patients receiving in-centre hemodialysis in this city during the novel coronavirus pandemic.

\section{Materials and methods}

We included patients receiving routine maintenance hemodialysis (MHD) at different healthcare facilities including university hospitals, state hospital, and private dialysis centers in Zonguldak city. Hospitalized and clinically confirmed cases were included. COVID-19 diagnosis was made based on clinical symptoms, radiologic findings on chest X-ray or computed tomography (CT) in addition to real-time polymerase chain reaction (RT-PCR) tests. The study was approved by the local Research Ethics Committee preceded by online approval of Turkish Ministry of Health.

In case of symptoms suggestive of coronavirus infection or in case of a definitive diagnosis of any patient or healthcare worker at any of the dialysis centres, even though asymptomatic we performed CT scans for all vulnerable patients and working staff followed by confirmation of highly suspicious cases by PCR or rapid antibody tests along with CBC, CRP, and biochemical blood tests. In our country, filiation [16] is performed throughout the COVID-19 pandemic. In our group, for each positive case, all individuals sharing the same dialysis session or had a close contact were screened primarily with a CT scan.

We considered any ground-glass opacity (GGO) as suspicious especially when multifocal and peripherally localized.
At the time of the study, CORADS criteria [17] was not routinely reported.

All patients with suspicious CT findings were hospitalized, tested with PCR, and treated when deemed fit. Due to difficulties in the obedience of social distancing and at home isolation recommendations for this vulnerable population, we hospitalized all patients who were radiologically and clinically suspected to have COVID-19.

Sociodemographic data such as age, gender, body mass index (BMI), hemodialysis initiation time, smoking history, etc., of all patients included in the study, were recorded. The primary causes of renal failure, comorbid conditions like diabetes, hypertension, chronic and occupational lung disease as well as the history of previous medications like ACE-I (Angiotensin-converting enzyme inhibitors) and ARB (Angiotensin receptor blockers) were questioned and recorded.

Complete blood count (Hemoglobin, Leukocyte, platelet, lymphocyte \& neutrophil counts), biochemical laboratory findings (C-reactive protein (CRP), urea, creatinine, D-dimer, troponin, lactate dehydrogenase (LDH), albumin, fibrinogen, lactate, sodium, potassium, calcium, phosphorus, parathormone, uric acid, and ferritin levels) as available were recorded at the time of diagnosis and tracked during the follow-up period.

The symptoms of COVID-19, diagnostic methods, thorax CT findings, PCR positivity and negativity intervals, intensive care admission, and need for intubation, duration, and response to medical treatments were recorded in detail.

All patients continued to receive standard hemodialysis treatment three times a week for $4 \mathrm{~h}$ per session. Existing vascular access routes were used and recorded. However, when mandatory as in the case of hypervolemia and hyperpotassemia, hemodialysis was applied more frequently. The dialysis machines were disinfected after each HD session using sodium hypochlorite solution according to the manufacturer's instruction.

Following the diagnosis of COVID-19, treatment was given to all patients in line with the recommendations of the Scientific Board of the Turkish Ministry of Health(MOH) [18]. All patients were started on hydroxychloroquine loading $2 * 400$ followed by $2 \times 200 \mathrm{mg}$ for a total of 5 days/ azithromycin $(500 \mathrm{mg}$ on the first day followed by 250 for a total of 5 days) plus oseltamivir $75 \mathrm{mg}$ given twice. Favipiravir was reserved for severe cases with dyspnea and $\mathrm{SpO} 2<90 \%$. It was given in the dose of $1600 \mathrm{mg}$ twice on day one followed by $600 \mathrm{mg}$ for the next 4 days.

Patients older than 50 years, those with comorbidities (cardiovascular diseases, diabetes mellitus (DM), hypertension (HT), cancer, chronic lung diseases, and other immunosuppressive conditions), those having criteria of severe pneumonia or respiratory distress, along with those with tachypnea or hypotension or $\mathrm{SpO} 2<92 \%$ and those with 
bilateral diffuse involvement in lung imaging were primarily managed as inpatients. Similarly, those who developed sepsis, septic shock, cardiomyopathy, arrhythmia or acute kidney injury and those developed poor prognostic criteria (blood lymphocyte count $<800 / \mu \mathrm{l}$ or serum CRP $>40 \mathrm{mg}$ / 1 or ferritin $>500 \mathrm{ng} / \mathrm{ml}$ or D-Dimer $>1000 \mathrm{ng} / \mathrm{ml}$, etc.) were also hospitalized on admission to hospital. Medical management of admitted patients continued as per the constantly updated guidelines and recommendations issued by Turkish MOH [18].

Patients' clinical evaluation, laboratory findings, and their response to medical treatment as well as a final state were evaluated thoroughly. The transmission rates of COVID-19 infection among healthcare professionals in contact with these patients and their medical management when needed were also evaluated. Scientific Package for Social Science (version 16.0; SPSS Inc., Chicago, IL, USA) was used for statistical analyses. We used Fisher's Exact test for nonparametric variables, independent-samples $T$ test for analyzing clinical and biochemical parameters. We also analyzed the risk factors for mortality using backward logistic regression of the Cox proportional hazard method. Differences were considered statistically significant for $\mathrm{P}$ values less than 0.05 .

\section{Results}

The study was carried out in 184 patients receiving maintenance hemodialysis treatment in the general state hospital, university hospital, and private dialysis center within the borders of the province of Zonguldak. A total of 34 hemodialysis patients were identified, hospitalized, and treated with a confirmed diagnosis of COVID-19. The frequency was found to be $18.4 \%$ in the HD population in the city center. 19 of these patients were females with a mean age of $62 \pm 13.2$ years. Mean dialysis duration was $66.9 \pm 57.7$ (min.1- max.204) months, mean BMI $26.1 \pm 5.4 \mathrm{~kg} / \mathrm{m}^{2}$ with an average hospital stay of $16.2 \pm 7.9$ (min.4- max.35) days. A total of 7 (20.5\%) patients had a smoking history, 4 of them were active smokers. None had a declared history of alcohol consumption.

As a comorbid disease, 20 (58.8\%) patients had hypertension, $19(55.8 \%)$ had diabetes, $15(44.1 \%)$ had the chronic obstructive pulmonary disease (COPD), 11 (32.3\%) had cardiovascular disease, 5 (14.7\%) had peripheral vascular disease, 2 had a history of malignancy (breast and thyroid cancer) and one had both epilepsy and chronic hepatitis B infection. Three (8.8\%) patients had a history of Coal workers' pneumoconiosis. Only one had no obvious comorbidity. Four patients were actively on either ACEI or ARB medication.
While at least one of the PCR measurements was positive in 32 patients, only 2 had negative results despite repetitive PCR measurements. However, antibody positivity was observed in 9 patients, including these two patients. Only 30 cases out of 34 were confirmed by the first specimen. One case had the second PCR positive for COVID-19 while the other was confirmed by the third specimen. Although first thorax CT findings were not fully compatible with 4 patients, the radiologic findings of the remaining 30 patients were consistent.

Even though 13 (38.2\%) patients tested positive on CT scans, they had no clinical symptoms. While fever was the first symptom in 12 (35.3\%) patients during the first evaluation, the cough was found to be the primary complaint in 5, fatigue in 1, and shortness of breath in another. Only one patient had nausea, vomiting, and diarrhea as a chief complaint.

The sociodemographic and laboratory data of patients are shown in Table 1. During the first application, leukopenia $(<3500)$ was found in 4 , neutropenia $(<2000)$ in 3 , and lymphopenia $(<1000)$ in $25(73.5 \%)$ patients. The absolute lymphocyte count was between 800 to 1000 in 5 patients, in the range of 501-800 in 11 while 9 had lymphocyte count less than 500. During the follow-up period, 8 patients developed leukopenia.

All patients received a triple Regimen consisting of hydroxychloroquine, azithromycin plus oseltamivir therapy on admission. Favipiravir was given to 9 patients. Azithromycin was discontinued in one case due to QT prolongation. None of the cases received tocilizumab, intravenous immunoglobulin (IVIG), nor immune plasma. Low molecular weight heparin (LMWH) was applied to 28 patients. Vascular access was native AVF in 33 patients while only one had tunneled catheter. No thromboembolic event was documented.

During the follow-up, two patients were treated with meropenem where one received ampicillin — sulbactam and another needed ceftriaxone as supportive treatment.

A total of 12 (35.3\%) patients were admitted to the intensive care unit where 7 of them required intubation. While one was extubated, the remaining $6(17.6 \%)$ died. When the data of patients admitted to the intensive care unit was analysed (Table 2), it was observed that they were relatively older $(p=0.04)$. The analysis of their laboratory data revealed that CRP and LDH levels were higher $(p=0.007$ and 0.035 respectively) while serum albumin and lymphocyte counts were much lower $(p=0.03,0.019$, and 0.001 , respectively). No significant difference was detected in terms of other data. When the data of patients deceased was compared to those recovered, patients who died were found to be older $(p=0.003)$ with higher levels of CRP and fibrinogen ( $p=0.023$ and 0.04 , respectively) in comparison to lower serum albumin and lymphocyte counts (respectively 
Table 1 Demographic and baseline laboratory data of all patients

\begin{tabular}{|c|c|c|c|}
\hline Parameters & All patients $(n: 34)$ & $\begin{array}{l}\text { Patients admitted to } \\
\text { ICU }(n: 12)\end{array}$ & Deceased patients $(n: 6)$ \\
\hline Age (years) & $62 \pm 13.2$ & $68 \pm 10.5$ & $72.8 \pm 6.6$ \\
\hline Gender (F/M) & $19 / 15$ & $6 / 6$ & $3 / 3$ \\
\hline Body mass index $\left(\mathrm{kg} / \mathrm{m}^{2}\right)$ & $26.1 \pm 5.4$ & $27 \pm 6.0$ & $27.6 \pm 6.8$ \\
\hline HD duration (months) & $66.9 \pm 57.7$ & $96 \pm 73.8$ & $108 \pm 90$ \\
\hline Hospitalization duration (days) & $16.2 \pm 7.9$ & $19 \pm 11$ & $12.3 \pm 11$ \\
\hline C-reactive protein $(\mathrm{mg} / \mathrm{dL})$ & $99 \pm 101$ & $163 \pm 95$ & $210 \pm 103$ \\
\hline Hemoglobin (g/dL) & $11.2 \pm 1.6$ & $11.3 \pm 1.0$ & $11.9 \pm 0.73$ \\
\hline Wight blood cell $\left(10^{6} / \mathrm{L}\right)$ & $5.858 \pm 2043$ & $5.690 \pm 2398$ & $6.798 \pm 2001$ \\
\hline Neutrophil $\left(10^{6} / \mathrm{L}\right)$ & $3887 \pm 1950$ & $4080 \pm 2212$ & $5461 \pm 2033$ \\
\hline Lymphocyte $\left(10^{6} / \mathrm{L}\right)$ & $913 \pm 575$ & $541 \pm 251$ & $566 \pm 228$ \\
\hline Platelet count $\left(10^{6} / \mathrm{L}\right)$ & $175,000 \pm 57,700$ & $169,900 \pm 75,400$ & $169,000 \pm 42,000$ \\
\hline Lactate $(\mathrm{mmol} / \mathrm{L})$ & $2.28 \pm 3.1$ & $2.94 \pm 4.16$ & $7.25 \pm 8.2$ \\
\hline Ferritin $(\mu \mathrm{g} / \mathrm{L})$ & $950 \pm 548$ & $749 \pm 630$ & $719 \pm 892$ \\
\hline D-dimer (ng/mL) & $775 \pm 604$ & $1032 \pm 810$ & $727 \pm 345$ \\
\hline Fibrinogen $(\mathrm{mg} / \mathrm{dL})$ & $431 \pm 134$ & $476 \pm 126$ & $518 \pm 94$ \\
\hline Albumin (mg/dL) & $3.6 \pm 0.5$ & $3.37 \pm 0.28$ & $3.3 \pm 0.23$ \\
\hline Creatinin kinase (U/L) & $120 \pm 90$ & $142 \pm 101$ & $212 \pm 45$ \\
\hline Troponin T (ng/L) & $0.20 \pm 0.29$ & $0.25 \pm 0.21$ & $0.30 \pm 0.30$ \\
\hline Creatinine (mg/dL) & $6.1 \pm 2.1$ & $5.5 \pm 1.6$ & $5.7 \pm 1.5$ \\
\hline Uric acid (mg/dl) & $5.9 \pm 2.1$ & $4.9 \pm 1.8$ & $3.8 \pm 1.98$ \\
\hline Lactate dehydrogenase (U/L) & $232 \pm 75$ & $280 \pm 105$ & $352 \pm 104$ \\
\hline Sodium $(\mathrm{mmol} / \mathrm{L})$ & $136 \pm 4.1$ & $135 \pm 4.9$ & $133 \pm 4.4$ \\
\hline Potassium (mmol/L) & $4.5 \pm 0.7$ & $4.4 \pm 0.6$ & $4.4 \pm 1.2$ \\
\hline Calcium (mg/dL) & $8.3 \pm 0.7$ & $8.3 \pm 0.8$ & $8.0 \pm 0.9$ \\
\hline Phosphorus (mg/dL) & $4.5 \pm 1.3$ & $4.1 \pm 1.4$ & $5.2 \pm 1.5$ \\
\hline Parathormone (ng/L) & $509 \pm 469$ & $456 \pm 335$ & $190 \pm 104$ \\
\hline
\end{tabular}

$p=0.025$ and 0.009 ) unlike that of the surviving group (Table 3). Age, baseline CRP, serum albumin, fibrinogen, neutrophil, and lymphocyte counts were analyzed using the Cox proportional hazard model backward stepwise LR (likelihood ratio) to identify independent risk factors of mortality. We found that only baseline CRP level (RR $1.013(95 \% \mathrm{Cl}$ 1.002-1.023) p:0.019) was a predictor of mortality.

In the same period, COVID-19 was diagnosed in a total of 13 healthcare professionals working in 3 dialysis centers where these cases emerged (11 females, mean age $44.4 \pm 5.8$ years), including two doctors, ten nurses in addition to one medical staff assistant. Ten of these cases were followed as outpatients with antibody positivity detected in all of them. The initial PCR test results were negative but confirmed with repetition in 6 out of 13 cases. The only case treated as inpatient had 3 consecutive PCR tests negative while hypoxemic with bilateral CT ground appearance had IgG antibodies to COVID 19 on day 14. Only one healthcare worker had hypertension as a comorbid disease. Six healthcare workers had CT findings while asymptomatic. On the other hand, six others were diagnosed with PCR performed because of their symptoms. One case was diagnosed primarily with consecutive PCR tests after applying with symptoms. Daily questioning of symptoms and control of body temperature was done. No medical prophylaxis was given to healthcare workers.

\section{Discussion}

In our study, the prevalence of COVID-19 in HD patients was found to be $18.4 \%$. While $35.3 \%$ of the diagnosed patients needed intensive care during their follow-up, the rate of mortality was $17.6 \%$. Almost all patients had at least one comorbidity. We found HT, DM, and COPD as the most common comorbid diseases in our cohort. $38.2 \%$ of our patients were basically suspected according to CT screening while asymptomatic. On the other hand, $35.3 \%$ had only fever as their first symptom. Although lymphopenia was the most common laboratory finding, a high CRP level was determined as the predictor of mortality.

COVID-19, a disease caused by a novel coronavirus, has become a major global human threat that has turned into a pandemic. Chronic kidney disease is associated with an 
Table 2 Data of ICU admitted and non-admitted Patients

\begin{tabular}{|c|c|c|c|}
\hline Parameters & $\begin{array}{l}\text { Patients with ICU } \\
\text { admission }(n: 12)\end{array}$ & $\begin{array}{l}\text { Patients w/o ICU } \\
\text { admission ( } n: 22)\end{array}$ & $P$ \\
\hline Age (years) & $68 \pm 10.5$ & $58.9 \pm 13.7$ & 0.04 \\
\hline Gender (F/M) & $6 / 6$ & $13 / 9$ & 0.72 \\
\hline BMI $\left(\mathrm{kg} / \mathrm{m}^{2}\right)$ & $27 \pm 6.0$ & $25.6 \pm 5.0$ & 0.51 \\
\hline HD duration (months) & $96 \pm 73.8$ & $47.5 \pm 33.9$ & 0.051 \\
\hline Hospitalization period (days) & $19 \pm 11$ & $14.7 \pm 4.9$ & 0.21 \\
\hline C-reactive protein (mg/dL) (baseline) & $163 \pm 95$ & $64.7 \pm 87.2$ & 0.007 \\
\hline C-reactive protein (mg/dL) (last visit) & $141 \pm 138$ & $23 \pm 24$ & 0.013 \\
\hline Hemoglobin (g/dl) (baseline) & $11.3 \pm 1.0$ & $11.1 \pm 1.9$ & 0.66 \\
\hline Hemoglobin (g/dl) (last visit) & $10.2 \pm 1.3$ & $9.9 . \pm 1.4$ & 0.62 \\
\hline White blood cell $\left(10^{6} / \mathrm{L}\right)$ (baseline) & $5690 \pm 2398$ & $5950 \pm 1876$ & 0.75 \\
\hline White blood cell $\left(10^{6} / \mathrm{L}\right)$ (last visit) & $10,800 \pm 11,200$ & $6500 \pm 2400$ & 0.21 \\
\hline Neutrophil $\left(10^{6} / \mathrm{L}\right)$ (baseline) & $4080 \pm 2212$ & $3782 \pm 1839$ & 0.69 \\
\hline Neutrophil ( $\left.10^{6} / \mathrm{L}\right)$ (last visit) & $8235 \pm 11,190$ & $4260 \pm 2088$ & 0.24 \\
\hline Lymphocyte $\left(10^{6} / \mathrm{L}\right)$ (baseline) & $541 \pm 251$ & $1116 \pm 604$ & 0.001 \\
\hline Lymphocyte $\left(10^{6} / \mathrm{L}\right)$ (last visit) & $935 \pm 429$ & $1320 \pm 644$ & 0.042 \\
\hline Platelet count $\left(10^{6} / \mathrm{L}\right)$ (baseline) & $169,900 \pm 75,400$ & $177,900 \pm 47,230$ & 0.74 \\
\hline Platelet count $\left(10^{6} / \mathrm{L}\right)$ (last visit) & $236,200 \pm 119,000$ & $190,300 \pm 61,350$ & 0.23 \\
\hline Ferritin ( $\mu \mathrm{g} / \mathrm{L})$ (baseline) & $749 \pm 630$ & $1045 \pm 498$ & 0.26 \\
\hline Ferritin ( $\mu \mathrm{g} / \mathrm{L})$ (last visit) & $1163 \pm 725$ & $1130 \pm 559$ & 0.90 \\
\hline D-dimer (ng/mL) (baseline) & $1032 \pm 810$ & $628 \pm 402$ & 0.12 \\
\hline D-dimer (ng/mL) (last visit) & $1371 \pm 1170$ & $1024 \pm 1281$ & 0.43 \\
\hline Fibrinogen (mg/dL) (baseline) & $476 \pm 126$ & $405 \pm 134$ & 0.14 \\
\hline Fibrinogen (mg/dL) (last visit) & $533 \pm 179$ & $453 \pm 178$ & 0.24 \\
\hline Lactate (mmol/L) (baseline) & $2.94 \pm 4.16$ & $1.4 \pm 0.66$ & 0.33 \\
\hline Albumin (mg/dL) (baseline) & $3.37 \pm 0.28$ & $3.7 \pm 0.55$ & 0.032 \\
\hline Creatinine kinase (U/L) (baseline) & $142 \pm 101$ & $103 \pm 82$ & 0.39 \\
\hline Troponin T (ng/L) (baseline) & $0.25 \pm 0.21$ & $0.17 \pm 0.34$ & 0.54 \\
\hline Creatinine (mg/dL) (baseline) & $5.5 \pm 1.6$ & $6.4 \pm 2.3$ & 0.21 \\
\hline Uric acid (mg/dL) (baseline) & $4.9 \pm 1.8$ & $6.6 \pm 2.16$ & 0.10 \\
\hline Lactate dehydrogenase (U/L) (baseline) & $280 \pm 105$ & $206 \pm 33$ & 0.035 \\
\hline
\end{tabular}

increased risk of both inpatient and outpatient pneumonia [9]. Moreover, the pneumonia-related mortality rate in CKD patients seems to be 14-16 times higher than in the general population [19]. Patients undergoing MHD have impaired immune system with increased susceptibility to infections resulting in a high rate of serious complications with an increased rate of mortality [13, 20]. Because of uremia related impairment of lymphocyte and granulocyte function, abnormal immune system response to COVID-19 infection may ensue [10]. In a recently published meta-analysis, CKD has been associated with increased severity of COVID-19 infection [21].

The estimated mortality of COVID-19 infection in the general population has been reported in the range of $1.4 \%-8 \%$ [1-3, 22-24]. Mortality risk increases significantly in patients requiring admission to the ICU, reported from 16 to $78 \%$ [22, 24-27]. In dialysis patients, mortality has been reported to be in the range of $16.2-31.3 \%$ [22, 26-29].
In our study, mortality was determined as $17.6 \%$ which is relatively low. This may be attributed to a variety of reasons. First, the average age in our group was lower (71 vs. 62 years) compared to the study from Spain where the rate of mortality was reported as $30.5 \%$ [28]. In another study involving hemodialysis patients reported from Wuhan, the origin of the pandemic, mortality was found to be $31.3 \%$ with the mean age reported being similar to our patient's population (63.3 vs 62 years) [29]. Another probable reason may be applying fillation all over our country. In $38 \%$ of our patients, the diagnoses were suspected early with help of CT scan even while many of them had no complaint. Thus, low mortality was achieved with early diagnosis, treatment, and close observation opportunities.

Comorbidities associated with increased mortality during COVID-19 are common among patients with CKD as notably observed in patients on maintenance hemodialysis therapy. With the recent increase in survival, the majority of 
Table 3 Data of living and deceased patients

\begin{tabular}{|c|c|c|c|}
\hline Parameters & Deceased patients $(n: 6)$ & Alive patients $(n: 28)$ & $P$ \\
\hline Age (years) & $72.8 \pm 6.6$ & $59.8 \pm 13.2$ & 0.003 \\
\hline Gender (F/M) & $3 / 3$ & $16 / 12$ & 0.79 \\
\hline BMI $\left(\mathrm{kg} / \mathrm{m}^{2}\right)$ & $27.6 \pm 6.8$ & $25.8 \pm 5.1$ & 0.57 \\
\hline HD duration (months) & $108 \pm 90$ & $56.6 \pm 43$ & 0.22 \\
\hline Hospitalization period (days) & $12.3 \pm 11$ & $17 \pm 7$ & 0.35 \\
\hline C-reactive protein (mg/dL) (baseline) & $210 \pm 103$ & $75.9 \pm 84.7$ & 0.023 \\
\hline C-reactive protein (mg/dL) (last visit) & $262 \pm 83$ & $22.8 \pm 22.6$ & 0.001 \\
\hline Hemoglobin (g/dl) (baseline) & $11.9 \pm 0.73$ & $11 \pm 1.7$ & 0.07 \\
\hline Hemoglobin (g/dl) (last visit) & $10.6 \pm 1.6$ & $9.9 \pm 1.3$ & 0.40 \\
\hline White blood cell $\left(10^{6} / \mathrm{L}\right)$ (baseline) & $6798 \pm 2001$ & $5657 \pm 2030$ & 0.24 \\
\hline White blood cell $\left(10^{6} / \mathrm{L}\right)$ (last visit) & $15,440 \pm 14,900$ & $6440 \pm 2190$ & 0.20 \\
\hline Neutrophil $\left(10^{6} / \mathrm{L}\right)$ (baseline) & $5461 \pm 2033$ & $3550 \pm 1793$ & 0.07 \\
\hline Neutrophil ( $\left.10^{6} / \mathrm{L}\right)$ (last visit) & $12,200 \pm 15,300$ & $4250 \pm 1900$ & 0.26 \\
\hline Lymphocyte $\left(10^{6} / \mathrm{L}\right)$ (baseline) & $566 \pm 228$ & $987 \pm 602$ & 0.009 \\
\hline Lymphocyte $\left(10^{6} / \mathrm{L}\right)$ (last visit) & $720 \pm 464$ & $1290 \pm 585$ & 0.03 \\
\hline Platelet count $\left(10^{6} / \mathrm{L}\right)$ (baseline) & $169,000 \pm 42,000$ & $176,000 \pm 61,000$ & 0.74 \\
\hline Platelet count $\left(10^{6} / \mathrm{L}\right)$ (last visit) & $245,000 \pm 159,000$ & $198,000 \pm 64,700$ & 0.50 \\
\hline Ferritin $(\mu \mathrm{g} / \mathrm{L})$ (baseline) & $719 \pm 892$ & $994 \pm 478$ & 0.58 \\
\hline Ferritin ( $\mu \mathrm{g} / \mathrm{L})$ (last visit) & $1118 \pm 859$ & $1148 \pm 574$ & 0.94 \\
\hline D-dimer (ng/mL) (baseline) & $727 \pm 345$ & $785 \pm 653$ & 0.76 \\
\hline D-dimer (ng/mL) (last visit) & $1475 \pm 1399$ & $1076 \pm 1216$ & 0.53 \\
\hline Fibrinogen (mg/dL) (baseline) & $518 \pm 94$ & $411 \pm 135$ & 0.04 \\
\hline Fibrinogen (mg/dL) (last visit) & $652 \pm 114$ & $448 \pm 172$ & 0.01 \\
\hline Lactate (mmol/L) (baseline) & $7.25 \pm 8.2$ & $1.45 \pm 0.71$ & 0.50 \\
\hline Albumin (mg/dL) (baseline) & $3.3 \pm 0.23$ & $3.65 \pm 0.52$ & 0.025 \\
\hline Creatinine kinase (U/L) (baseline) & $212 \pm 45$ & $108 \pm 89$ & 0.11 \\
\hline Troponin T (ng/L) (baseline) & $0.30 \pm 0.30$ & $0.19 \pm 0.29$ & 0.69 \\
\hline Creatinine (mg/dL) (baseline) & $5.7 \pm 1.5$ & $6.1 \pm 2.2$ & 0.57 \\
\hline Uric acid (mg/dL) (baseline) & $3.8 \pm 1.98$ & $6.19 \pm 2.0$ & 0.31 \\
\hline Lactate dehydrogenase(U/L) (baseline) & $352 \pm 104$ & $207 \pm 32$ & 0.019 \\
\hline
\end{tabular}

HD patients are elderly but have certain comorbid conditions like hypertension, diabetes, cardiovascular, and lung disease along with an underlying immune-compromised state. Altogether may contribute in varying proportions to worse outcomes, especially in patients suffering from COVID-19 [14, $30,31]$. Comorbid diseases were observed in all but one of our patients. The most notable comorbidities in our group were hypertension, diabetes, cardiovascular, and chronic obstructive pulmonary diseases. The incidence of chronic obstructive pulmonary disease was higher in our patients compared to other studies. [28, 29]. Compared to the data reported by Xiong $\mathrm{F}$. et al., smoking rates (including exsmokers) were found to be lower in our patients $(20.5 \% \mathrm{vs}$. 29.8) [29]. Zonguldak city, where our study was conducted, is one of the most important mining centres in Turkey. Majority of people in this city work in mines. In a previous study investigating COPD in Zonguldak, a prevalence of $11.1 \%$ was found in adults of the general population with a higher (14.1\%) prevalence in those over 40 years [15].
In another study from Turkey also, COPD prevalence was reported as $2.5 \%$ [32].

The most common clinical symptoms reported were fever, cough, sputum production, fatigue, nausea, vomiting, and diarrhea. [3, 4, 28, 29]. Prevalence of fever has been reported in the range of 51.9-98\% [3, 28]. Many of our patients were asymptomatic since they were diagnosed early. In our study, fever and cough are the most common clinical symptoms, compatible with the literature but with a relatively lower rate.

Various laboratory parameters like elevated interleukin (IL)-6, LDH, troponin I, CRP, fibrinogen, D-Dimer, and ferritin levels along with lymphopenia were reported in patients with COVID-19 disease. Prolonged prothrombin time (PT) and active partial thromboplastin time (aPTT) were observed in severe cases [3, 33]. Patients on MHD have disrupted immune systems making them more vulnerable to infections $[13,20]$. Lymphopenia is common in patients with COVID19 infection and might be a critical factor associated with 
disease severity and mortality in general [34]. Another study showed that a high CRP level at presentation was associated with the risk of death [28]. Lymphopenia was present in the majority of our patients. In addition, we found that CRP and fibrinogen levels were higher while serum albumin and lymphocyte counts were lower in our deceased patients. We found that baseline CRP level was the only predictor of mortality in our patients.

Hemodialysis patients are at high risk of being infected with COVID-19 entailing additional preventive measures and strict rules in managing the epidemic in hemodialysis centres. Priority for dialysis centers is early recognition of patients, isolation of those with probable symptoms, and dialyzing them in a separate session or in a room away from other patients. Meanwhile, ensuring protective equipment used by healthcare workers should be considered [6].

Currently, in accordance with Center for Disease Control and Prevention (CDC)'s guidance, it is recommended that patients with symptoms should be directed to hospitalization rather than being dialyzed with isolation in outpatient hemodialysis facilities [35]. In our study, following the first case detection, patients were screened and diagnosed while asymptomatic. In addition, all patients were hospitalized and dialyzed in separate equipped rooms and halls away from other patients. An additional 14 days isolation period was applied following discharge when eligible.

In general, optimal treatment for COVID-19 disease is still controversial. The indication for antiviral therapy is uncertain with no proved drug for the treatment of SARSCoV-2 infection till the moment [36]. The number of randomized controlled trials performed with different treatment protocols is limited so far. In a randomized controlled trial with Remdesivir, the clinical benefit has not been demonstrated [37]. In another randomized controlled study, the efficacy of Favipiravir was investigated [38]. In this Study, favipiravir treatment did not improve the clinical recovery rate on Day 7 when compared to Arbidol treatment. A posthoc analysis found that Favipiravir was superior to arbidol in terms of the clinical recovery rate at day 7 in patients with moderate illness [38]. Hydroxychloroquine, oseltamivir, and azithromycin was started in our patients according to the recommendations initially published by our Ministry of Health. Favipiravir was added to the treatment of nine patients. At the time of study in March, contrary to later versions last of which issued in October 2020 suggesting favipiravir as an outpatient choice when relevant, It was reserved for severe cases with dyspnea and hypoxemia as second-line treatment. LMWH was added to the treatment in line with the updated MOH's recommendations. Therefore, LMWH was not used in all patients. Hydroxychloroquine showed higher in-vitro activity against SARS-CoV-2 and has been used in the treatment of admitted COVID-19 patients. It has been reported that the use of hydroxychloroquine either alone or in combination with azithromycin reduces the viral load of COVID-19 in comparison with the control group as shown by repeated measurements [39]. South Korea and China have drafted guidelines for the use of Hydroxychloroquine and chloroquine for the therapeutic treatment of COVID-19 [33].

Our study also has a number of limitations. First, the retrospective character of our study. In addition, some of the patients were asymptomatic and identified by Thorax CT screening. Third, some laboratory tests such as IL-6, procalcitonin were not done in all patients. Finally, the interpretation of our findings might be limited by the small sample size.

In conclusion, the COVID-19 prevalence is high in HD patients in this study. The frequency of comorbid diseases is high in the dialysis population. Early screening of asymptomatic patients on maintenance dialysis therapy sharing in the same session with a positive case may increase the possibility of early diagnosis and consequently resulting in lower mortality. Along with that, immediate treatment with hydroxychloroquine may be an important factor that resulted in a lower mortality rate. In symptomatic patients, fever was found to be the most common sign. The rate of lymphopenia observed in the hemodialysis community is high. High CRP level at the time of diagnosis has been determined as the predictor of mortality. For Hemodialysis patients highly suspected of COVID-19, as CT findings may precede PCR positivity, we believe that adding radiologic evaluation with CT scan in the workup will aid in early detection and accordingly limiting the spread of the disease in this special group.

Acknowledgements We thank both Dr. Zeynep Mehpare Eskici M.D. and Staff nurse Betul Turkoglu, affiliation supplemented, for their support and great efforts in collecting and recording clinical and laboratory findings and preparing for analysis. Zeynep M. Eskici, M.D., Department of Biochemistry, Zonguldak State Hospital, Zonguldak, Turkey. Betul Turkoglu, Patient's Pre-dialysis Education Program manager, Zonguldak state Hospital, Zonguldak, Turkey

Author contributions Research idea and study design: MI, YK; data acquisition: MI, YO; data analysis/interpretation: YK,MI; statistical analysis: YK; supervision and mentorship: YK. Each author contributed important intellectual content during manuscript drafting and revision and agrees to be personally accountable for the individual's own contributions and to ensure that questions pertaining to the accuracy or integrity of any portion of the work, even one in which the author was not directly involved, are appropriately investigated and resolved, including with documentation in the literature if appropriate.

Funding The authors declare that they have no relevant financial interests.

\section{Compliance with ethical standards}

Conflict of interest None of the authors have any conflict of interest related to this article.

Form of consent Not applicable. 
Support None of the authors had any support.

\section{References}

1. Zhu N et al. (2020) A novel coronavirus from patients with pneumonia in China, 2019.

2. Mahase E (2020) Coronavirus: covid-19 has killed more people than SARS and MERS combined, despite lower case fatality rate. 2020, British Medical Journal Publishing Group.

3. Huang $\mathrm{C}$ et al (2020) Clinical features of patients infected with 2019 novel coronavirus in Wuhan. China 395(10223):497-506

4. Naicker $\mathrm{S}$ et al. (2020) The novel coronavirus 2019 epidemic and kidneys [published online ahead of print March 7, 2020].

5. Basile C et al. (2020) Recommendations for the prevention, mitigation and containment of the emerging SARS-CoV-2 (COVID19) pandemic in haemodialysis centres. 35(5): 737-741.

6. Perico L, Benigni A, Remuzzi GJN (2020) Should COVID19 concern nephrologists? Why and to what extent? Emerging Impasse Angiotensin Blockade 144(5):213-221

7. Organization WH (2020) Report of the WHO-China joint mission on coronavirus disease 2019 (COVID-19). https://www.who.int/ docs/default-source/coronaviruse/who-china-joint-mission-oncovid-19-final-report.pdf. Geneva.

8. Ward RA (1995) Heparinization for routine hemodialysis. Adv Ren Replace Ther 2(4):362-370

9. Chou C-Y et al. (2014) Risk of pneumonia among patients with chronic kidney disease in outpatient and inpatient settings: a nationwide population-based study. 93(27).

10. Zhou J et al. (2017) Human intestinal tract serves as an alternative infection route for Middle East respiratory syndrome coronavirus. 3(11): eaao4966

11. Wang HJKM (2020) Maintenance Hemodialysis and Coronavirus Disease 2019 (COVID-19): Saving Lives With Caution, Care, and Courage

12. Syed-Ahmed M, Narayanan MJAickd (2019) Immune dysfunction and risk of infection in chronic kidney disease. 26(1): 8-15.

13. Betjes MGJNRN (2013) Immune cell dysfunction and inflammation in end-stage renal disease. 9(5): 255.

14. Guan W-j et al. (2020) Clinical characteristics of coronavirus disease 2019 in China. 382(18): 1708-1720.

15. Örnek T et al. (2015) Prevalence of chronic obstructive pulmonary disease in Zonguldak Province of Turkey [Zonguldak il merkezinde kronik obstrüktif akciğer hastalığı prevelansı]

16. Demirtas T, Tekiner H (2020) Filiation: a historical term the COVID-19 outbreak recalled in Turkey. Erciyes Med J 42(3):354-359

17. Prokop M et al. (2020) CO-RADS-A categorical CT assessment scheme for patients with suspected COVID-19: definition and evaluation. Radiology 201473.

18. Health, T.M.o., COVID-19 (SARS-CoV-2 Infection) Guideline 9 october 2020, workup of Scientific Board. available from https:// covid19.saglik.gov.tr/Eklenti/39061/0/covid-19rehberieriskinhast atedavisipdf.pdf

19. Sarnak MJ, BLJC (2001) Jaber, Pulmonary infectious mortality among patients with end-stage renal disease. 120(6): 1883-1887

20. Vaziri ND et al. (2012) Effect of uremia on structure and function of immune system. 22(1): 149-156
21. Henry BM, Lippi GJIu, nephrology, Chronic kidney disease is associated with severe coronavirus disease 2019 (COVID-19) infection. 2020: 1-2.

22. Grasselli G et al (2020) Baseline characteristics and outcomes of 1591 patients infected with SARS-CoV-2 admitted to ICUs of the Lombardy region. Italy 323(16):1574-1581

23. Yi Y et al. (2020) COVID-19: what has been learned and to be learned about the novel coronavirus disease. 16(10): 1753.

24. Ma Y et al. (2019) novel coronavirus disease in hemodialysis (HD) patients: Report from one HD center in Wuhan, China. 2020.

25. Madjid M et al. (2020) Potential effects of coronaviruses on the cardiovascular system: a review.

26. Alberici F et al. (2020) Management of patients on dialysis and with kidney transplant during SARS-COV-2 (COVID-19) pandemic in Brescia, Italy.

27. Scarpioni $\mathrm{R}$ et al (v) Covid-19 and its impact on nephropathic patients: the experience at Ospedale" Guglielmo da Saliceto" in Piacenza. 37(2): 1-5.

28. Goicoechea, M., et al., COVID-19: Clinical course and outcomes of 36 maintenance hemodialysis patients from a single center in Spain. 2020.

29. Xiong F et al. (2020) Clinical Characteristics of and Medical Interventions for COVID-19 in Hemodialysis Patients in Wuhan, China

30. Wynants L et al. (2020) Prediction models for diagnosis and prognosis of covid-19 infection: systematic review and critical appraisal. 369(m1328): 1.

31. Guo T et al. (2020) Cardiovascular implications of fatal outcomes of patients with coronavirus disease 2019 (COVID-19)

32. Barış SA et al (2011) Prevalence of smoking and chronic obstructive pulmonary disease amongst teachers working in Kocaeli, Turkey. Multidisciplinary Respiratory Med 6(2):92

33. Kaul DJCMR and Practice (2020) An overview of coronaviruses including the SARS-2 coronavirus-Molecular biology, epidemiology and clinical implications

34. Xu Z et al. (2020) Pathological findings of COVID-19 associated with acute respiratory distress syndrome. 8(4): 420-422.

35. Ikizler TAJAJoKD (2020) COVID-19 and dialysis units: what do we know now and what should we do?

36. Organization WH (2020) Clinical management of severe acute respiratory infection (SARI) when COVID-19 disease is suspected: interim guidance, 13 March 2020. 2020, World Health Organization.

37. Wang Y et al. (2020) Remdesivir in adults with severe COVID-19: a randomised, double-blind, placebo-controlled, multicentre trial.

38. Chen $\mathrm{C}$ et al. Favipiravir versus arbidol for COVID-19: a randomized clinical trial. 2020.

39. Gautret $P$ et al. (2020) Hydroxychloroquine and azithromycin as a treatment of COVID-19: results of an open-label non-randomized clinical trial: 105949 .

Publisher's Note Springer Nature remains neutral with regard to jurisdictional claims in published maps and institutional affiliations. 